\title{
Usefulness of Spectral Analysis of Respiratory Rate Variability to Help in Pediatric Sleep Apnea-Hypopnea Syndrome Diagnosis
}

\author{
Verónica Barroso-García*, Gonzalo C. Gutiérrez-Tobal, Member, IEEE, Leila Kheirandish-Gozal, \\ Daniel Álvarez, Fernando Vaquerizo-Villar, Félix del Campo, David Gozal, and Roberto Hornero, \\ Senior Member, IEEE
}

\begin{abstract}
The sleep apnea-hypopnea syndrome (SAHS) is a chronic respiratory disorder of high prevalence among children (up to $4 \%$ ). Nocturnal polysomnography (PSG) is the gold standard method to diagnose SAHS, which is a complex, expensive, and time-consuming test. Consequently, alternative simplified methods are demanded. We propose the analysis of the respiratory rate variability (RRV) signal, directly obtained from the airflow (AF) signals. The aim of our study is to evaluate the usefulness of the spectral information obtained from RRV in the diagnosis of pediatric SAHS. A database composed of $946 \mathrm{AF}$ and blood oxygen saturation $\left(\mathrm{SpO}_{2}\right)$ recordings from children between 0 and 13 years old was used. Our database was divided into four severity groups according to the apnea-hipopnea index (AHI): no-SAHS (AHI $<1$ events/h), mild (1 events/h $\leq$ AHI $<5$ events/h), moderate (5 events/h $\leq$ AHI $<10$ events/h), and severe SAHS (AHI $\geq 10$ events/h). RRV and $3 \%$ oxygen desaturation index $\left(\mathrm{ODI}_{3}\right)$ were obtained from $\mathrm{AF}$ and $\mathrm{SpO}_{2}$ recordings, respectively. A spectral band of interest was determined $(0.09-0.20 \mathrm{~Hz}$.) and a total of 12 spectral features were extracted. Nine of these features showed statistically significant differences $(p$-value $<$ 0.05) among the four severity groups. The spectral features from RRV along with $\mathrm{ODI}_{3}$ were used as inputs to binary logistic regression (LR) classifiers. The diagnostic performance of LR models were evaluated for the AHI cut-off points of 1, 5, and $10 \mathrm{e} / \mathrm{h}$, achieving $66.5 \%, 84.0 \%$, and $88.5 \%$ accuracy, respectively. These results outperformed those obtained by single $\mathrm{ODI}_{3}$. The joint use of the spectral information from RRV and $\mathrm{ODI}_{3}$ achieved a high diagnostic capability in the most severely-affected children, thus showing their complementarity. These results suggest that the information contained in RRV spectrum together with $\mathrm{ODI}_{3}$ is useful to help identify moderate-to-severe SAHS.
\end{abstract}

* This work was supported by 'Ministerio de Ciencia, Innovación y Universidades' and 'European Regional Development Fund (FEDER)' under projects DPI2017-84280-R and RTC-2017-6516-1, and by 'European Commission' and 'FEDER' under project 'POCTEP 0378_AD_EEGWA_2_P'.

V. Barroso-García was in a receipt of a 'Ayuda para financiar la contratación predoctoral de personal investigador' grant from the Consejería de Educación de la Junta de Castilla y León and the European Social Fund. F. Vaquerizo-Villar was in receipt of a 'Ayuda para contratos predoctorales para la Formación de Profesorado Universitario (FPU)' grant from the Ministerio de Educación, Cultura y Deporte (FPU16/02938). L. Kheirandish-Gozal and D. Gozal were supported by National Institutes of Health (NIH) grant HL130984.

V. Barroso-García, G. C. Gutiérrez-Tobal, F. Vaquerizo-Villar, and R. Hornero, are with the Biomedical Engineering Group, Universidad de Valladolid, Spain (e-mail: veronica.barroso@gib.tel.uva.es).

D. Álvarez and F. del Campo are with the Hospital Universitario Río Hortega of Valladolid, Spain (e-mail: fsas@telefonica.net).

L. Kheirandish-Gozal and D. Gozal are with the Department of Child Health, The University of Missouri School of Medicine, Columbia, Missouri, USA (email: gozald@health.missouri.edu).

\section{INTRODUCTION}

The sleep apnea-hypopnea syndrome (SAHS) is a prevalent respiratory disorder that affects up to $4 \%$ of pediatric population [1]. Children affected by SAHS suffer from repeated apnea (airflow absence) and hipopnea (airflow reduction) episodes while sleeping [2]. This disease may lead to serious medical consequences, affecting cardiovascular and central nervous systems, as well as cognitive development of children [1], [2]. Consequently, it is essential for SAHS to be detected and treated timely.

The gold standard test to diagnose childhood SAHS is inhospital overnight polysomnography (PSG) [3]. PSG involves monitoring and recording multiple biomedical signals from pediatric subjects during sleep, like electroencephalogram (EEG), electrocardiogram (ECG), photoplethysmography (PPG), airflow (AF), and blood oxygen saturation $\left(\mathrm{SpO}_{2}\right)$ [3]. The recordings from PSG are used to compute the apnea-hypopnea index (AHI), which is the number of apnea and hypopnea events per hour of sleep (e/h) [4]. Pediatric SAHS and its severity are diagnosed according to AHI [4]. Despite PSG effectiveness, this test is complex, expensive, time-consuming and especially uncomfortable for children [5], [6].

These limitations, together with the high prevalence of the disease in children, have increased the demand for simpler diagnostic techniques. The analysis of a reduced set of signals is commonly used for this purpose. ECG, PPG, $\mathrm{SpO}_{2}$, and $\mathrm{AF}$, have been widely analyzed in the pediatric SAHS context [7]-[11]. The analysis of respiratory rate variability (RRV) is proposed in our study. RRV have been successfully used to assist in diagnosing SAHS in both adults and children [11], [12]. However, the study of its spectral content has not been fully addressed. RRV, defined as the elapsed time between consecutive complete respiratory cycles, is directly obtained from AF [13]. This time varies when apnea and hipopnea events occur [11], [12]. Consequently, the repeated occurrence of apneic episodes modifies RRV signal in frequency domain [11], [12], which naturally leads us to its analysis. In addition, 3\% oxygen desaturation index $\left(\mathrm{ODI}_{3}\right)$ obtained from $\mathrm{SpO}_{2}$ has been incorporated into the study for comparison purposes, since it is a widely used clinical parameter to diagnose pediatric SAHS [14], [15].

According to the aforementioned considerations, the recurrence of apneas and hypopneas alters the RRV spectrum [11], [12]. We hypothesized that the spectrum of RRV contains useful information to assist in the pediatric SAHS 
diagnosis. Consequently, the objective of our study was to evaluate the usefulness of the information derived from RRV spectrum to diagnose SAHS in children. In order to reach this goal, several spectral features have been derived from the power spectrum of the RRV signal, due to the utility that they have already shown in the SAHS context [11], [12]. The usefulness of the spectral features extracted from RRV, as well as their complementarity with $\mathrm{ODI}_{3}$, were assessed by logistic regression (LR) modelling. The AHI cut-off points commonly used to determine the different pediatric SAHS severity degrees were evaluated.

\section{SUBJECTS AND SIGNALS}

Nine hundred and forty six pediatric subjects suspected of suffering from SAHS participated in this study. All of them underwent PSG in the Pediatric Sleep Unit at the Comer Children's Hospital of the University of Chicago. The study protocol was approved by the Ethics Committee of the Hospital and the legal caretakers of children gave their informed consent. The pediatric subjects were diagnosed according to the rules of the American Academy of Sleep Medicine (AASM) [4]. The common AHI cut-off points 1, 5, and $10 \mathrm{e} / \mathrm{h}$ were evaluated [16]. The population under study was randomly allocated in two groups: training (60\%) and test (40\%), to design and validate the proposed methodology, respectively. Table I shows the demographic and clinical data of the subjects under study. No significant differences ( $p$ value $>0.05$ ) were found in age, gender, body mass index (BMI), and AHI between the training group and the test group, after applying the non-parametric Mann-Whitney test.

PSG was conducted using a digital polysomnography system (Polysmith, Nihon Kohden America Inc., Irvine, CA, USA). All recordings included in our dataset had more than 3 hours of total sleep time. AF recordings, acquired with a thermistor and sampled at $100 \mathrm{~Hz}$, were used to obtain RRV signals. AF recordings were normalized following the methodology suggested by Varady et al. to minimize differences among children due to age [17]. Moreover, AF artifacts were discarded using a comparison of the standard deviation and the kurtosis of AF segments. RRV was computed as the time between consecutive inspiratory onsets [13]. We looked for the points that are relative maximum in $\mathrm{AF}$ (the points where the first derivative changes from positive to negative) and the elapsed time between consecutive maximum points was computed to obtain RRV [13]. Afterwards, an interpolation process (resampling at 100 $\mathrm{Hz}$ ) was required prior to the spectral analysis since the RRV samples do not follow a constant sampling rate [11], [12]. $\mathrm{SpO}_{2}$, used to obtain $O D I_{3}$, was recorded by means of an oximeter at the sample rate of $25 \mathrm{~Hz}$. Artifacts presented in $\mathrm{SpO}_{2}$ were removed following the methodology suggested in others studies [10], [15].

\section{METHODS}

\section{A. Spectral Analysis}

The recurrence of apneas and hypopneas causes alterations in RRV spectrum [11], [12]. Consequently, we proceeded to estimate the power spectral density (PSD) of each interpolated RRV signal using the Welch method (Hamming window of $2^{16}$ samples, i.e. $\approx 655$ seconds, $50 \%$ overlap, and length of the discrete Fourier transform of $2^{17}$ points) [18].

Fig.1 shows the normalized PSDs averaged for the four SAHS severity groups in the training set: no-SAHS (AHI $<1$ $\mathrm{e} / \mathrm{h})$, mild SAHS $(1 \mathrm{e} / \mathrm{h} \leq \mathrm{AHI}<5 \mathrm{e} / \mathrm{h})$, moderate SAHS (5 $\mathrm{e} / \mathrm{h} \leq \mathrm{AHI}<10 \mathrm{e} / \mathrm{h})$, and severe SAHS (AHI $\geq 10 \mathrm{e} / \mathrm{h})$.

Differences among the four SAHS severity groups in the training dataset were evaluated using the non-parametric Mann-Whitney test in order to obtain the spectral bands of interest [12], [14]. As can be seen in Fig. 2, the spectral band of interest corresponds to the region that shows a greater tendency to reach statistically significant differences ( $p$-value $<0.05$ after Bonferroni correction) between the SAHS severity groups: $0.09-0.20 \mathrm{~Hz}$. The spectral band of interest, obtained as indicated below, can also be observed in Fig. 1.

TABLE I. DEMOGRAPHIC AND CLINICAL DATA OF THE SUBJECTS UNDER STUDY

\begin{tabular}{|l|c|c|c|}
\hline & All & $\begin{array}{c}\text { Training } \\
\text { group }\end{array}$ & Test group \\
\hline Subjects (n) & 946 & 570 & 376 \\
\hline Age (years) & $6[6]$ & $6[5]$ & $6[6]$ \\
\hline Males (n) & $584(61.73 \%)$ & $339(59.47 \%)$ & $245(65.16 \%)$ \\
\hline BMI (kg/m2) & $17.92[6.17]$ & $17.72[6.74]$ & $18.07[6.01]$ \\
\hline AHI (e/h) & $3.82[7.80]$ & $4.17[8.34]$ & $3.33[6.44]$ \\
\hline $\mathbf{N}^{\mathbf{0}}$ with AHI $\geq \mathbf{1}(\mathbf{n})$ & $783(82.77 \%)$ & $479(84.04 \%)$ & $304(80.85 \%)$ \\
\hline $\mathbf{N}^{\mathbf{0}}$ with $\mathbf{A H I} \geq \mathbf{5}(\mathbf{n})$ & $397(41.97 \%)$ & $256(44.91 \%)$ & $141(37.5 \%)$ \\
\hline $\mathbf{N}^{\mathbf{0}}$ with $\mathbf{A H I} \geq \mathbf{1 0}(\mathbf{n})$ & $225(23.78 \%)$ & $145(25.44 \%)$ & $80(21.28 \%)$ \\
\hline
\end{tabular}

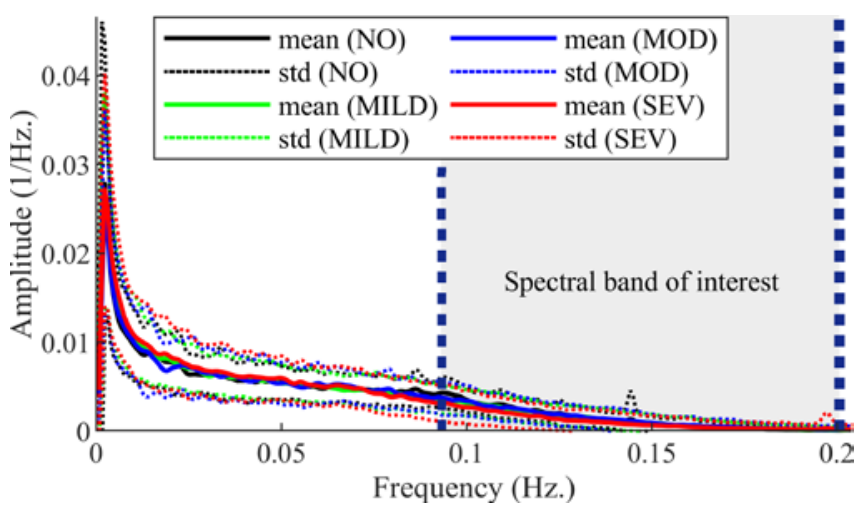

Figure 1. Mean and standard deviation (std) of power spectral density (PSD) of the four severity groups: no-SAHS (NO), mild (MILD), moderate (MOD), and severe (SEV) SAHS, and spectral band of interest.

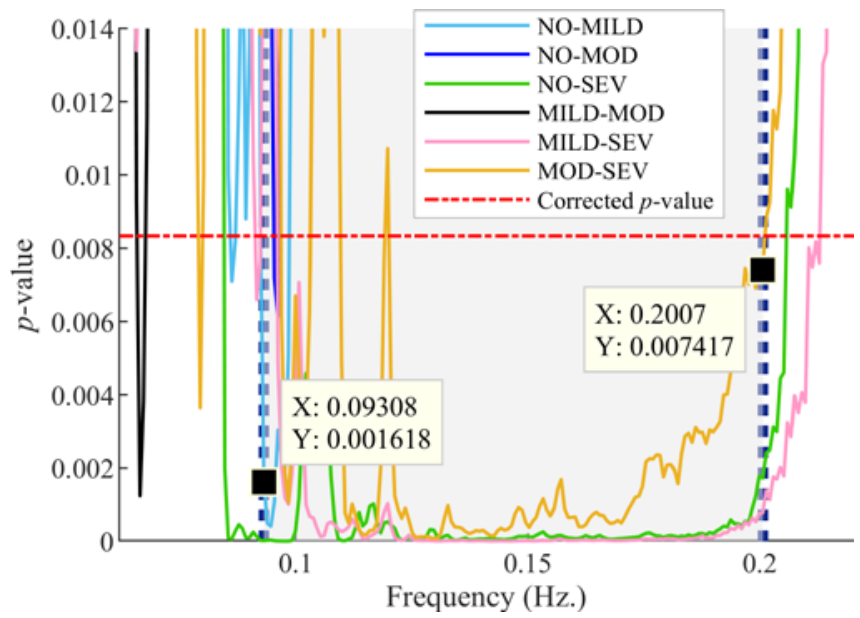

Figure 2. $\quad p$-values versus frequency for each of the comparisons between SAHS severity groups: NO vs. MILD, NO vs. MOD, NO vs. SEV, MILD vs. MOD, MILD vs. SEV, MOD vs. SEV. 
In order to study the RRV spectrum, the following features were extracted:

- $\quad$ Median frequency (MF) of the full RRV spectrum. This measure, defined as the frequency component under which $50 \%$ of the cumulative power is obtained, allows to estimate the spectral power concentration [10].

- $\quad$ Maximum and minimum amplitude (MA, $m A)$ of PSD in the spectral band of interest [10], [14].

- $\quad$ First-to-fourth statistical moments $\left(M f_{1}-M f_{4}\right)$ and median were computed to evaluate the central tendency, dispersion, asymmetry, and concentration of the spectral information in the band of interest obtained [12], [14].

- $\quad$ Spectral entropy ( $\left.S E_{1}\right)$, quadratic spectral entropy $\left(S E_{2}\right)$, and cubic spectral entropy $\left(S E_{3}\right)$ were obtained to measure the flatness of the RRV spectrum and quantify the signal irregularity [11].

- Wootters' distance (WD). WD is a disequilibrium measurement that allows to measure the RRV irregularity [10], [12].

\section{B. Oximetry index}

$\mathrm{ODI}_{3}$ was obtained from $\mathrm{SpO}_{2}$ the recordings. The algorithm used for computing $\mathrm{ODI}_{3}$ is based in the study developed by Taha et al. [19].

\section{Logistic Regression}

LR is a standard method for binary classification [20]. It estimates the posterior probability of belonging to the positive or negative class according to the predictor variables, i.e. the input features to the model [20]. The logistic function follows the expression:

$$
\pi(x)=\frac{e^{\beta_{0}+\beta_{1} x_{1}+\ldots+\beta_{k} x_{k}}}{1+e^{\beta_{0}+\beta_{1} x_{1}+\ldots+\beta_{k} x_{k}}},
$$

where $\pi(x)$ is the posterior probability of membership to the SAHS class, $\beta_{0}$ is the interceptor, $\beta_{i}(i=1, \ldots, k)$ are the coefficients associated to each predictor variable, and $k$ the number of features in the LR model. $\beta_{0}$ and $\beta_{i}$ were optimized using the algorithm of maximum likelihood estimation [20].

\section{Statistical Analysis}

The spectral features extracted from RRV did not pass the Lilliefors normality test. For this reason, the nonparametric Kruskal-Wallis test was used to assess the existence of statistically significant differences ( $p$-value < 0.05 after Bonferroni correction) in the obtained features from the four severity groups. The non-parametric MannWhitney test was used to evaluate possible significant differences ( $p$-value $<0.05$ after Bonferroni correction) between each pair of SAHS severity groups. The diagnostic performance of the LR models was evaluated in terms of sensitivity (Se), specificity (Sp), and accuracy (Acc).

\section{RESUlts}

\section{A. Training group}

Table II shows the median and interquartile range (IQR) values of the spectral features obtained for each SAHS severity group, as well as their corresponding $p$-values after
Bonferroni correction. All of the features, except $M F, S E_{2}$, and $S E_{3}$, obtained statistically significant differences ( $p$ value $<0.05)$ among groups. Three LR classifiers were trained with the 12 spectral features from $R R V\left(L R^{R R V}\right)$, using the AHI cut-offs 1,5 , and $10 \mathrm{e} / \mathrm{h}$. Moreover, these features along with the $\mathrm{ODI}_{3}$ fed three more LR classifiers $\left(\mathrm{LR}^{\mathrm{RRV}, O D I 3}\right)$.

\section{B. Test group}

The diagnostic performance obtained by $\mathrm{LR}^{\mathrm{RRV}}$ and

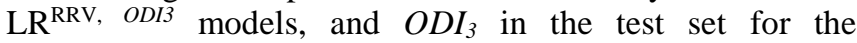
thresholds 1,5 , and $10 \mathrm{e} / \mathrm{h}$, is shown in table III. Binary

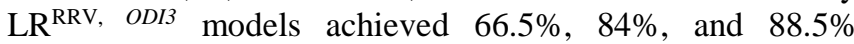
accuracy for 1,5 , and $10 \mathrm{e} / \mathrm{h}$, respectively, outperforming the results obtained with single $\mathrm{ODI}_{3}$ and $\mathrm{LR}^{\mathrm{RRV}}$.

\section{DISCUSSION AND CONCLUSIONS}

In this work, the usefulness of RRV signal to help in SAHS diagnosis in children has been evaluated. A spectral analysis of RRV was carried out for this purpose. A new spectral band of interest $(0.09-0.20 \mathrm{~Hz})$ was defined by means of statistical techniques, which have already shown their usefulness in previous studies of pediatric SAHS [10], [14]. Afterwards, this band was characterized by spectral features ( $M A, m A, M f_{1}-M f_{4}$, median, and $B P$ ). $M F, S E_{1}, S E_{2}$, $S E_{3}$, and $W D$ features were also calculated to complete the

TABLE II. VALUES OF SPECTRAL FEATURES FOR THE FOUR SAHS SEVERITY GROUPS

\begin{tabular}{|c|c|c|c|c|c|}
\hline \multirow{2}{*}{$\begin{array}{l}\text { Spectral } \\
\text { Features }\end{array}$} & No-SAHS & Mild SAHS & $\begin{array}{c}\text { Moderate } \\
\text { SAHS }\end{array}$ & $\begin{array}{l}\text { Severe } \\
\text { SAHS }\end{array}$ & \multirow{2}{*}{$p$-value } \\
\hline & $\begin{array}{c}\text { Median } \\
\text { [IQR] }\end{array}$ & $\begin{array}{c}\text { Median } \\
\text { [IQR] }\end{array}$ & $\begin{array}{c}\text { Median } \\
{[I Q R]}\end{array}$ & $\begin{array}{c}\text { Median } \\
{[I Q R]}\end{array}$ & \\
\hline MF $\left(10^{-2}\right)$ & 4.50 [1.79] & 4.35 [1.66] & $4.50[1.96]$ & $3.89[2.31]$ & 0.058 \\
\hline$M A\left(10^{-3}\right)$ & $5.28[2.03]$ & $4.71[1.98]$ & $4.75[2.10]$ & $4.30[3.46]$ & $<0.05$ \\
\hline$m A\left(10^{-6}\right)$ & 3.36 [23.15] & 3.06 [39.78] & 2.69 [68.40] & $0.17[7.60]$ & $<0.05$ \\
\hline$M f_{1}\left(10^{-3}\right)$ & $1.23[0.86]$ & $1.22[0.96]$ & $1.25[1.22]$ & 0.89 [1.38] & $<0.05$ \\
\hline$M f_{2}\left(10^{-3}\right)$ & $1.35[0.52]$ & $1.25[0.66]$ & $1.26[0.69]$ & $1.05[1.03]$ & $<0.05$ \\
\hline$M f_{3}\left(10^{0}\right)$ & $1.18[0.93]$ & 1.04 [1.07] & $1.10[1.24]$ & 1.50 [1.27] & $<0.05$ \\
\hline$M f_{4}\left(10^{0}\right)$ & 3.48 [3.20] & 3.18 [2.69] & 3.37 [3.34] & $4.48[4.59]$ & $<0.05$ \\
\hline Median $\left(10^{-3}\right)$ & $0.49[1.03]$ & 0.58 [1.31] & 0.42 [1.65] & $0.16[1.04]$ & $<0.05$ \\
\hline$S E_{1}\left(10^{-1}\right)$ & $9.05[0.51]$ & $9.09[0.56]$ & $9.05[0.71]$ & $8.89[0.74]$ & $<0.05$ \\
\hline$S E_{2}\left(10^{-1}\right)$ & 7.91 [1.24] & 7.92 [1.34] & 7.90 [1.16] & $7.72[1.18]$ & 0.404 \\
\hline$S E_{3}\left(10^{-1}\right)$ & $6.45[2.28]$ & $6.35[2.10]$ & 6.40 [1.94] & $6.32[1.65]$ & 0.864 \\
\hline$W D\left(10^{-1}\right)$ & 3.90 [1.08] & 3.79 [1.32] & 3.95 [1.63] & $4.22[1.61]$ & $<0.05$ \\
\hline
\end{tabular}

TABLE III. DIAGNOSTIC PERFORMANCE OF LR MODELS AND ODI $\mathrm{I}_{3}$ FOR AHI CUT-OFF 1, 5, AND $10 \mathrm{E} / \mathrm{H}$

\begin{tabular}{|c|c|c|c|}
\hline & \multicolumn{3}{|c|}{ AHI cut-off $=1 \mathrm{e} / \mathrm{h}$} \\
\hline & $\mathrm{Se}(\%)$ & Sp (\%) & Acc (\%) \\
\hline $\mathbf{L R}^{\mathbf{R R V}}$ & 55.3 & 44.4 & 53.2 \\
\hline $\mathbf{L R}^{\mathrm{RRV}, \text { ODI3 }}$ & 65.5 & 70.8 & 66.5 \\
\hline \multirow[t]{3}{*}{$\mathrm{ODI}_{3}$} & 59.9 & 86.1 & 64.9 \\
\hline & \multicolumn{3}{|c|}{ AHI cut-off $=5 \mathrm{e} / \mathrm{h}$} \\
\hline & $\mathrm{Se}(\%)$ & $S p(\%)$ & Acc (\%) \\
\hline $\mathbf{L R}^{\mathbf{R R V}}$ & 52.5 & 66.4 & 61.2 \\
\hline $\mathbf{L R}^{\mathrm{RRV}, \mathrm{ODI3}}$ & 74.5 & 89.8 & 84.0 \\
\hline \multirow[t]{3}{*}{$\mathrm{ODI}_{3}$} & 69.5 & 89.4 & 81.9 \\
\hline & \multicolumn{3}{|c|}{ AHI cut-off $=10 \mathrm{e} / \mathrm{h}$} \\
\hline & Se (\%) & $S p(\%)$ & Acc (\%) \\
\hline $\mathbf{L R}^{\mathbf{R R V}}$ & 52.5 & 68.6 & 65.2 \\
\hline $\mathbf{L R}^{\mathrm{RRV}, \mathrm{ODI3}}$ & 78.8 & 90.9 & 88.3 \\
\hline $\mathrm{ODI}_{3}$ & 81.3 & 88.5 & 87.0 \\
\hline
\end{tabular}


spectral information from RRV. Statistically significant differences were observed in 9 out of the 12 extracted features, showing the usefulness of these features. In this way, a LR model fed with both the spectral features from RRV and the $\mathrm{ODI}_{3}$ reached moderate-to-high accuracies (66.5\%, 84\%, and 88.3\%) for 1, 5, and 10 e/h, respectively. Therefore, the joint use of the spectral information from RRV and $\mathrm{ODI}_{3}$ was able to achieve high diagnostic capability in the most severely affected children, showing their complementarity.

Several studies have evaluated the use of a reduced set of biomedical signals to detect SAHS in children, commonly assessing a single cut-off. Shouldice et al. [7] analyzed temporal and spectral features from 50 ECGs. They used a quadratic discriminant analysis (QDA) classifier, reaching 84.0\% Acc (85.7\% Se and 81.8\% Sp) for the threshold $1 \mathrm{e} / \mathrm{h}$. Lázaro et al. [8] analyzed 21 PPGs. In their study, a linear discriminant analysis (LDA) classifier was used, obtaining 86.7\% Acc (100\% Se and 71.4\% Sp) for 5 e/h. Garde et al. [9] analyzed spectral and temporal features from $146 \mathrm{SpO}_{2}$ and PRV signals. They used LDA and the AHI cut-off $5 \mathrm{e} / \mathrm{h}$, achieving $84.9 \%$ Acc (88.4\% Se and $83.6 \%$ Sp). The results of these studies are barely generalizable due to a low number of subjects involved in them. By contrast, we have evaluated our methodology according to the three common cut-off points that establish the SAHS severity degrees, and validated our proposal with a large database.

Some studies have already evaluated their methodologies according to the different degrees of SAHS severity. In this regard, Barroso-García et al. [11] analyzed the variability and irregularity of $501 \mathrm{AF}$ and RRV signals. The LR models used in their study achieved 60\% Acc for 1 e/h (60.5\% Se and $58.6 \% \mathrm{Sp}$ ), $76 \%$ Acc for 5 e/h (65\% Se and $80.6 \% \mathrm{Sp}$ ), and $80 \%$ Acc for 10 e/h (83.3\% Se and 79\% Sp). Hornero et al. [10] used a multi-layer perceptron neural network fed with features from $4191 \mathrm{SpO}_{2}$ recordings. Their study reached $75.2 \%$ Acc (84\% Se and 53.2\% Sp), 81.7\% Acc (68.2\% Se and 87.2\% Sp), and 90.2\% Acc (68.7\% Se and $94.1 \% \mathrm{Sp}$ ) for cut-off points 1, 5, and $10 \mathrm{e} / \mathrm{h}$, respectively. However, both studies reported lower diagnostic performance in $5 \mathrm{e} / \mathrm{h}$. By contrast, our results for this cut-off were higher. Pediatric subjects with an AHI $\geq 5 \mathrm{e} / \mathrm{h}$ have a high risk of being affected by major adverse health consequences and morbidities [1], [16]. Moreover, surgical treatment with adenotonsillectomy is recommended when $\mathrm{AHI} \geq 5 \mathrm{e} / \mathrm{h}$ [1], [16]. Hence, their early diagnosis is crucial and an automatic detection could help in these cases.

This study has some limitations. Although the number of subjects is high, an even larger database would reinforce the general character of our results. In addition, RRV signal has been analyzed only in the frequency domain, thus it could be interesting to conduct complementary analysis in the time domain. Using methods of multiclass classification or AHI estimation could be also useful, which constitutes another interesting future research line.

In summary, a band of interest in RRV spectrum, with significant differences between the pediatric SAHS severity groups, was determined. RRV signal was characterized by means of spectral features. The LR model fed with these features and $\mathrm{ODI}_{3}$ reached high diagnostic ability for AHI cut-off values $5 \mathrm{e} / \mathrm{h}$ and $10 \mathrm{e} / \mathrm{h}$, outperforming the individual diagnostic capability of $\mathrm{ODI}_{3}$ in all thresholds. These results suggest that the information contained in RRV spectrum combined with $\mathrm{ODI}_{3}$ is useful to help identify moderate-tosevere pediatric SAHS.

\section{REFERENCES}

[1] A. G. Kaditis et al., "Obstructive sleep disordered breathing in 2- to 18year-old children: diagnosis and management,” Eur. Respir. J., vol. 47, no. 1, pp. 69-94, 2016.

[2] C. L. Marcus et al., "Diagnosis and management of childhood obstructive sleep apnea syndrome.,” Pediatrics, vol. 130, no. 3, pp. 576-84, 2012.

[3] C. Jon, "Polysomnography in Children," in Pediatric Otolaryngology for the Clinician, Totowa, NJ: Humana Press, 2009, pp. 35-47.

[4] R. B. Berry et al., "Rules for Scoring Respiratory Events in Sleep: Update of the 2007 AASM Manual for the Scoring of Sleep and Associated Events,” J. Clin. Sleep Med., vol. 08, no. 05, pp. 597-619, Oct. 2012.

[5] P. J. Ryan et al., "Validation of British Thoracic Society guidelines for the diagnosis of the sleep apnoea/hypopnoea syndrome: can polysomnography be avoided?,” Thorax, vol. 50, no. 9, pp. 972-975, Sep. 1995.

[6] E. S. Katz, R. B. Mitchell, and C. M. D’Ambrosio, “Obstructive Sleep Apnea in Infants,” Am. J. Respir. Crit. Care Med., vol. 185, no. 8, pp. 805816, 2012.

[7] R. B. Shouldice, L. M. O’Brien, C. O’Brien, P. de Chazal, D. Gozal, and C. Heneghan, "Detection of Obstructive Sleep Apnea in Pediatric Subjects using Surface Lead Electrocardiogram Features,” Sleep, vol. 27, no. 4, pp. 784-792, Jun. 2004.

[8] J. Lazaro, E. Gil, J. M. Vergara, and P. Laguna, "Pulse Rate Variability Analysis for Discrimination of Sleep-Apnea-Related Decreases in the Amplitude Fluctuations of Pulse Photoplethysmographic Signal in Children,” IEEE J. Biomed. Heal. Informatics, vol. 18, no. 1, pp. 240-246, 2014.

[9] A. Garde, P. Dehkordi, W. Karlen, D. Wensley, J. M. Ansermino, and G. A. Dumont, "Development of a Screening Tool for Sleep Disordered Breathing in Children Using the Phone Oximeter ${ }^{\mathrm{TM}}$," PLoS One, vol. 9, no. 11, p. e112959, Nov. 2014.

[10] R. Hornero et al., "Nocturnal Oximetry-based Evaluation of Habitually Snoring Children,” Am. J. Respir. Crit. Care Med., vol. 196, no. 12, pp. 1591-1598, 2017.

[11] V. Barroso-García et al., "Irregularity and variability analysis of airflow recordings to facilitate the diagnosis of paediatric sleep apnoea-hypopnoea syndrome,” Entropy, vol. 19, no. 9, 2017.

[12] G. C. Gutierrez-Tobal, R. Hornero, D. Álvarez, J. V Marcos, and F. del Campo, "Linear and nonlinear analysis of airflow recordings to help in sleep apnoea-hypopnoea syndrome diagnosis,” Physiol. Meas., vol. 33, no. 7, pp. 1261-1275, 2012.

[13] D. Cysarz, R. Zerm, H. Bettermann, M. Frühwirth, M. Moser, and M. Kröz, "Comparison of Respiratory Rates Derived from Heart Rate Variability, ECG Amplitude, and Nasal/Oral Airflow,” Ann. Biomed. Eng. vol. 36, no. 12, pp. 2085-2094, 2008.

[14] G. C. Gutiérrez-Tobal, M. L. Alonso-Álvarez, D. Álvarez, F. del Campo, J. Terán-Santos, and R. Hornero, "Diagnosis of pediatric obstructive sleep apnea: Preliminary findings using automatic analysis of airflow and oximetry recordings obtained at patients' home,” Biomed. Signal Process. Control, vol. 18, pp. 401-407, 2015.

[15] U. J. Magalang and J. Dmochowski, "Prediction of the Apnea-Hypopnea Index From Overnight Pulse Oximetry,” CHEST J., vol. 124, no. 5, pp. 1694-1701, 2003.

[16] M. Luz Alonso-Álvarez et al., "Consensus document on sleep apneahypopnea syndrome in children,” Arch. Bronconeumol., vol. 47, no. SUPPL. 5, pp. 2-18, 2011.

[17] P. Várady, T. Micsik, S. Benedek, and Z. Benyó, "A novel method for the detection of apnea and hypopnea events in respiration signals," IEEE Trans. Biomed. Eng., vol. 49, no. 9, pp. 936-942, 2002.

[18] P. D. Welch, "The Use of Fast Fourier Transform for the Estimation of Power Spectra: A Method Based on Time Aver. aging Over Short, Modified Periodograms,” 1967.

[19] B. H. Taha et al., "Automated Detection and Classification of SleepDisordered Breathing From Conventional Polysomnography Data," Sleep, vol. 20, no. 11, pp. 991-1001, Nov. 1997.

[20] D. W. Hosmer and S. Lemeshow, Applied Logistic Regression. London, UK: John Wiley \& Sons, 2000. 